\title{
Bioestimulantes derivados de Ascophyllum nodosum associados ao glyphosate nas caracteristicas agronômicas da soja $\mathbf{R R}^{\circledR}$
}

\author{
Biostimulants derived from Ascophyllum nodosum associated of \\ glyphosate in agronomic characteristics of soybean $R R^{\circledast}$
}

Christiano Lima Lobo de Andrade ${ }^{1}$, Alessandro Guerra da Silva ${ }^{1}$, Gabriel Bressiani Melo², Ranier Vieira Ferreira ${ }^{1}$, Isabela Cristina Sousa Moura ${ }^{1}$, Gabriel Gonçalves Costa Siqueira ${ }^{1}$

Resumo - O glyphosate é o principal herbicida no controle de plantas daninhas na soja. Porém, sintomas de fitointoxicação após a aplicação desse herbicida são relatados na soja $R^{\circledR}$. Neste contexto os bioestimulantes podem potencializar as atividades metabólicas da planta em função dos biorreguladores, aminoácidos e nutrientes presentes em sua formulação, tornando-se uma alternativa para minimizar os efeitos fitotóxicos do glyphosate. Dessa forma o objetivo deste trabalho foi o conhecer os efeitos do uso de bioestimulantes, derivados de Ascophyllum nodosum, associados ao herbicida glyphosate nos incrementos das características agronômicas da cultura da soja. Para isso, conduziu-se um experimento na safra 2014/15 em Montividiu-GO com treze tratamentos, resultantes

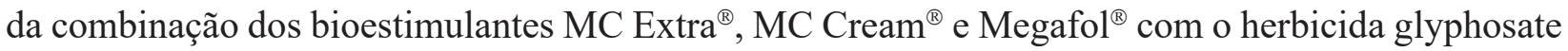
(Roundup Transorb ${ }^{\circledR}$ ). Os resultados permitiram concluir que a aplicação conjunta, no estádio de cinco trifólios desenvolvidos, do herbicida com o MC Extra ${ }^{\circledR}$ e com o Megafol ${ }^{\circledR}$ proporcionaram maiores incrementos na produtividade de grãos da soja. Já o MC Cream ${ }^{\circledR}$ proporcionou maior produtividade de grãos aos quinze dias após a aplicação do glyphosate. Assim, os bioestimulantes comprovaram o potencial de uso na cultura da soja.

Palavras-chave: fitotoxicidade, Glycine max, herbicidas, injúrias, produtividade

Abstract - Glyphosate is the main herbicide in weed control in soybean. However, phytotoxicity symptoms after application of this herbicide are reported in $\mathrm{RR}^{\circledR}$ soybean. In this context, biostimulants can potentiate the metabolic activities of the plant in function of the bioregulators, amino acids and nutrients present in its formulation, becoming an alternative to minimize the phytotoxic effects of glyphosate. Thus, the objective of this work was to identify the use of biostimulants, derived from Ascophyllum nodosum, associated to the herbicide glyphosate in the increments of the agronomic characteristics of the soybean crop. For this, an experiment was conducted in the 2014/15 harvest in Montividiu-GO with thirteen treatments, resulting from the combination of biostimulants MC Extra $^{\circledR}, \mathrm{MC} \mathrm{Cream}^{\circledR}$ and $\mathrm{Megafol}^{\circledR}$ with the herbicide glyphosate (Roundup Transorb ${ }^{\circledR}$ ). The results allowed us to conclude that the five-year combined application of the herbicide with MC Extra ${ }^{\circledR}$

Recebido: Dezembro 15, 2017. Aceito: Outubro 09, 2018.

${ }^{1}$ Programa de Pós-graduação em Produção Vegetal, Faculdade de Agronomia, Universidade de Rio Verde - UNIRV, Fazenda fontes do saber, caixa postal 104, CEP 75909-104, Rio Verde, GO, Brasil. E-mail: christiano.instrutoria@gmail.com; silvaag@yahoo.com.br; raniervieiraferreira@hotmail.com; isabelamourapsi@gmail.com; gabrielgoncalvescsiqueira@gmail.com; guilhermebrag@gmail.com

${ }^{2}$ Programa de Pós-graduação em Ciências Agrárias, Instituto Federal Goiano Campus Rio Verde - IFG, Rod. Sul Goiana Km, 01, Zona Rural, CEP 75906-896, Rio Verde, GO, Brasil. E-mail: gabremelo@gmail.com 
and Megafol ${ }^{\circledR}$ provided greater increases in grain yield of soybean. MC Cream ${ }^{\circledR}$ provided higher grain yield at 15 days after application of glyphosate. Thus, the biostimulants proved the potential of use in the soybean crop.

Keywords: phytotoxification, Glycine max, herbicides, injuries, yield

\section{Introdução}

A soja (Glycine max) é considerada uma commodity agrícola por ter seus preços controlados pelo mercado mundial, sendo a principal oleaginosa cultivada no mundo (United States, 2018). Neste contexto, o Brasil se destaca entre os maiores produtores, ocupando a segunda posição no ranking mundial (Brasil, 2018a). Além disto, no Brasil, a soja é cultura agrícola com maior crescimento de área cultivada nas últimas três décadas, obtendo acréscimo de $49 \%$ neste período (Brasil, 2018b).

Um dos fatores limitantes ao cultivo de soja no Brasil é o manejo das plantas daninhas, principalmente devido ao surgimento de espécies resistentes ocasionado pelas sucessivas aplicações de herbicidas (López-Ovejero et al., 2006; Beckie, 2011). Em função da interferência imposta à soja, principalmente pela competição por água, nutrientes e luz, as plantas daninhas comprometem o crescimento inicial da cultura e o desenvolvimento de estruturas reprodutivas (Gava, 2014). Isto, consequentemente, irá comprometer o desenvolvimento de características agronômicas da soja, ocasionando reduções de produtividade.

Por auxiliar no manejo de plantas daninhas, um dos marcos na sojicultora brasileira foi a liberação do cultivo da soja Roundup Ready ${ }^{\circledR}$ (RR). Esta inovação trouxe uma nova ferramenta ao manejo de plantas daninhas, proporcionando a oportunidade de rotacionar uma molécula com amplo espectro de ação (Gazziero, 2006). Isto permitiu o aumento da eficiência de controle e a redução do impacto ambiental causado pelo uso excessivo dos latifolicidas (Gazziero, 2006; Zobiole et al., 2010; Merotto et al., 2015).

Apesar disso, com a seleção de biótipos de plantas daninhas tolerantes ao glyphosate, tornou-se comum o aumento das doses aplicadas deste ingrediente ativo e aplicações seqüenciais do herbicida (Ferreira et al., 2013). Entretanto, com o uso itensivo do glyphosate em pós-emergência da soja, passou a ser comum a observação de sintomas de amerelecimento das folhas superiores, conhecido como yellow flashing (Santos et al., 2007; Zablotowicz e Reddy, 2007). Este efeito é causado pelo ácido aminometilfosfônico (AMPA), primeiro metabólito fitotóxico do glyphosate, que causa redução da biomassa seca da raiz e da parte aérea, além de redução dos teores de clorofila (Merotto et al., 2015).

Além disso, o glyphosate tem alta afinidade com cátions bi e trivalente, o que confere ao herbicida propriedade quelante a elementos como cobre, ferro, manganês e zinco, causando sua imobilização e reduzindo a capacidade fotossintética da soja (Coutinho e Mazo, 2005). Por outro lado, o uso do glyphosate pode causar redução da fixação biológica de nitrogênio, pois as bactérias fixadoras de N (Bradyrhizobium spp.) apresentam uma forma sensível da enzima EPSPS (Procópio et al., 2004; Dvoranen et al., 2008; Zobiole et al., 2012).

Uma possibilidade de amenizar os efeitos do glyphosate é o uso de bioestimulantes, por ser substâncias aplicadas com o objetivo de melhorar a eficiência nutricional e a tolerância a estresses abióticos (Du Jardin, 2015). Estes efeitos são atribuídos à presença de biorreguladores e outras substâncias na composição dos produtos, como os macro e micronutrientes, aminoácidos, oligossacarídeos, citocininas, auxinas, ácido abscísico, giberelinas, betaínas e alginatos (Mackinnon et al., 2010; Sharma et al., 2012; Povero et al., 2016). Dessa forma, estes compostos 
podem alterar, inibir ou modificar processos bioquímicos nas plantas, causando diferentes respostas fisiológicas, podendo conferir maior tolerância a ação fitotóxica do herbicida (Van Oosten et al., 2017).

A definição industrial de bioestimulante foi inicialmente proposta em 2012 visando padronizar e classificar tais substâncias em função a matéria prima e dos benéficos gerados por sua aplicação. (Vandenkoornhuyse et al., 2015; Van Oosten et al., 2017). Durante um longo periodo, seus efeitos foram considerados duvidosos devido a incerteza quanto a sua composição quimica e à dificuldade em determinar quais componentes específicos estavam promovendo contribuições positivas às plantas (Du Jardin, 2015).

Dentre os bioestimulantes utilizados na agricultura estão os derivados da alga Ascophyllum nodosum (L.), originária dos mares árticos e atlântico norte sob condições ambientais extremas (Craigie, 2011; Khan et al., 2012). A gama de compostos presentes no extrato de alga possuem funções no metabolismo da soja além da potencialização dos sinais químicos, podendo auxiliar a planta em superar condições climáticas e de manejo adversas (Sharma et al., 2012). Dessa forma, a habilidade da soja em competir com plantas daninhas e sua capacidade de recuperação das injúrias causadas pelo glyphosate, pode ser incrementada pela utilização destes compostos, garantindo vantagens na síntese de fotoassimilados e enchimento de grãos.

Diante disto, o trabalho tem como objetivo conhecer as possibilidades de usos de bioestimulantes derivados de Ascophyllum nodosum associados a aplicação de glyphosate para promoverem incrementos nas características agronômicas da cultura da soja.

\section{Material e métodos}

O experimento foi instalado a campo (17 $11^{\circ}$ '02,3" $\mathrm{S}$; $51^{\circ} 12^{\prime} 52,9^{\prime \prime} \mathrm{W}$ a $894 \mathrm{~m}$ de altitude) no município de Montividiu-GO na safra 2014/15, em solo cultivado no sistema de plantio direto. O clima na localidade é do tipo Aw, sendo caracterizado como clima tropical com estação seca, apresentando chuvas mais intensas no verão em relação ao inverno (Köppen). Os dados de precipitação e temperatura média do ar durante a condução do experimento estão apresentados na Figura 1.

A amostra de solo da área experimental foi coletada na camada de 0-20 cm de profundidade, tendo as seguintes características físico-químicas: pH em $\mathrm{CaCl}_{2}$ : 5,3; Ca: 3,01; $\mathrm{Mg}$ : 0,74; $\mathrm{Al}: 0,1$; $\mathrm{H}+\mathrm{Al}: 4,0 ; \mathrm{K}: 0,3 \mathrm{em} \mathrm{cmol}_{\mathrm{c}} \mathrm{dm}^{-3} ; \mathrm{P}: 3,6 \mathrm{mg} \mathrm{dm}^{-3}$; CTC: $8,0 \mathrm{cmol}_{\mathrm{c}} \mathrm{dm}^{-3}$, V: $50,52 \%$; MO: $30,10 \mathrm{~g} \mathrm{~kg}^{-1}$,

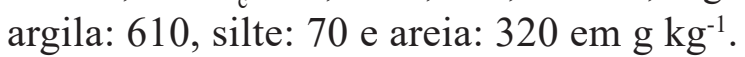

$\mathrm{O}$ delineamento utilizado foi o de blocos casualizados com quatro repetições, sendo avaliados treze tratamentos, levando -se em consideração a aplicação de glyphosate em pós-emergência da soja (Tabela 1). Todos os bioestimulantes utilizados são derivados da alga Ascophyllum nodosum. Os produtos utilizados foram: MC Cream ${ }^{\circledR}$ (Carbono orgânico, Mn: $15 \mathrm{~g} \mathrm{~L}^{-1}$; Zn: $5 \mathrm{~g} \mathrm{~L}^{-1}$ ), MC Extra ${ }^{\circledR}$ (Carbono orgânico: $200 \mathrm{~g} \mathrm{~L}^{-1}$; N: $10 \mathrm{~g} \mathrm{~L}^{-1}$; $\mathrm{K}_{2} \mathrm{O}: 200 \mathrm{~g} \mathrm{~L}^{-1}$ ) e Megafol ${ }^{\circledR}$ (Carbono orgânico: 109,8 g L L$^{-1}$; $\left.\mathrm{N}: 36,6 \mathrm{~g} \mathrm{~L}^{-1} ; \mathrm{K}_{2} \mathrm{O}: 97,6 \mathrm{~g} \mathrm{~L}^{-1}\right)$. Para as aplicações de glyphosate, foi utilizado o produto comercial Roundup Transorb ${ }^{\circledR}$ (Concentração $580 \mathrm{~g} \mathrm{~L}^{-1}$; suspensão concentrada) na dose de 900 g e.a. ha-1, dose esta recomendada para o controle de plantas

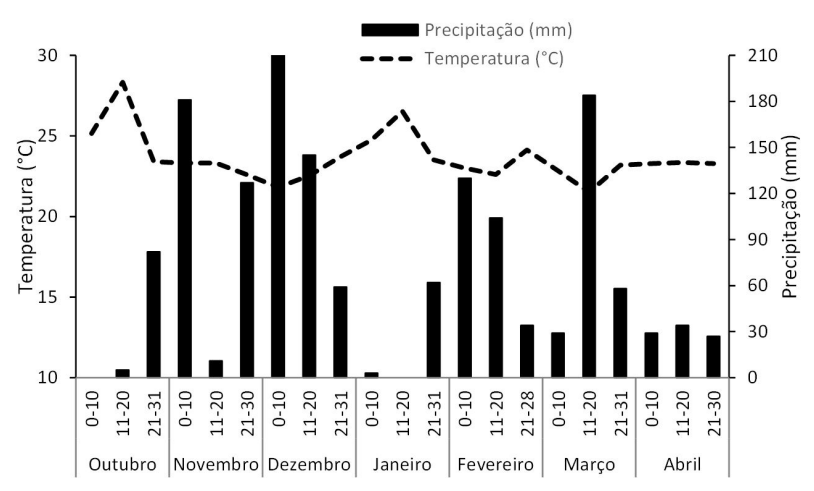

Figura 1. Valores médios de precipitação (mm) e de temperatura $\left({ }^{\circ} \mathrm{C}\right)$ durante a condução do ensaio. Montividiu (GO), safra 2014/15. 
Tabela 1. Tratamentos do experimento de bioestimulantes derivados de Ascophyllum nodosum associados ao glyphosate nas características agronômicas da soja $\mathrm{RR}^{\circledR}$. Montividiu-GO, 2014/15.

\begin{tabular}{|c|c|c|}
\hline & $1^{\text {a }}$ Aplicação (Dose - L ou kg p.c. ha' $\left.{ }^{-1}\right)^{1 /}$ & $2^{\mathrm{a}}$ Aplicação (Dose - L ou kg p.c. ha ${ }^{-1}$ / época) \\
\hline $\mathrm{T}_{1}$ & MC Extra $(1,0)$ & - \\
\hline $\mathrm{T}_{2}$ & MC Extra $(1,0)+$ glyphosate & - \\
\hline $\mathrm{T}_{3}^{2}$ & Megafol $(1,0)+$ glyphosate & - \\
\hline $\mathrm{T}_{4}$ & MC Extra $(1,0)+$ glyphosate & Megafol $(1,0) / \mathrm{R}_{5}$ \\
\hline $\mathrm{T}_{5}^{4}$ & MC Extra $(0,5)+$ Megafol $(1,0)+$ glyphosate & - \\
\hline $\mathrm{T}_{6}$ & MC Extra $(0,5)+$ glyphosate & MC Extra $(0,5) / R_{5}$ \\
\hline $\mathrm{T}_{7}$ & MC Cream $(1,0)+$ glyphosate & - \\
\hline $\mathrm{T}_{8}$ & MC Cream $(1,0)+$ glyphosate & MC Cream $(1,0) / \mathrm{R}_{5}$ \\
\hline $\mathrm{T}_{9}$ & MC Cream $(1,0)+$ glyphosate & MC Extra $(1,0) / \mathrm{R}_{5}$ \\
\hline $\mathrm{T}_{10}$ & MC Extra $(1,0)+$ glyphosate & MC Cream $(1,0) / \mathrm{R}_{5}$ \\
\hline $\mathrm{T}_{11}$ & glyphosate & MC Cream $(1,0) / \mathrm{R}_{1}$ \\
\hline $\mathrm{T}_{12}$ & glyphosate & MC Extra $(1,0) / \mathrm{R}_{1}$ \\
\hline $\mathrm{T}_{13}$ & glyphosate & - \\
\hline
\end{tabular}

1/ Aplicação realizada em pós-emergência da soja no estádio $\mathrm{V}_{5}$. Aplicação de glyphosate realizada na dose de 900 g e.a. ha ${ }^{-1}$.

daninhas em pós-emergência (Brasil, 2018b). Para os tratamentos que coincidiram a mesma época de aplicação do bioestimulante com o glyphosate, utilizou-se ambos os produtos na mesma calda de aplicação.

As unidades experimentais continham seis linhas de 10,0 $\mathrm{m}$ de comprimento, espaçadas em 0,5 $\mathrm{m}$. A área útil foi obtida levando-se em consideração as duas linhas centrais, desconsiderando $0,5 \mathrm{~m}$ de cada extremidade, apresentando, portanto $9 \mathrm{~m}^{2}$. Uma semana antes da semeadura da soja, foi realizada a dessecação de manejo, de forma mecanizada, com aplicação de glyphosate na dose de $1.200 \mathrm{~g}$ e.a ha ${ }^{-1}$. Um dia antes da semeadura, foi aplicado à lanço, 250 e $150 \mathrm{~kg} \mathrm{ha}^{-1}$ dos fertilizantes superfosfato simples e cloreto de potássio, respectivamente.

A semeadura da soja foi realizada em 29 de outubro de 2014. A cultivar empregada foi a BMX Desafio $\mathrm{RR}^{\circledR}$, caracterizada por ter hábito de crescimento indeterminado, ciclo precoce com grupo de maturação 7.3 para a microrregião do experimento, além de ter flor branca e pubescência cinza. Os tratos culturais foram realizados de acordo com os recomendados para a cultura, procedendo ao controle de pragas e doenças para não interferir nas características analisadas (Bueno et al., 2014). Todos os tratamentos foram manejados com capina manual para evitar a interferência das plantas daninhas no desenvolvimento das plantas.

As aplicações foram realizadas com pulverizador costal pressurizado por $\mathrm{CO}_{2}$, munido de barra com seis pontas tipo TT 110-02, de leque duplo e volume de calda equivalente a $150 \mathrm{~L} \mathrm{ha}^{-1}$ quando regulado a 2,5 $\mathrm{kgf} \mathrm{cm}^{-2}$. Após os recipientes de 2,0 L estarem abastecidos com água, foi adicionado o herbicida e/ou o bioestimulante, sendo promovido a agitação da calda antes da aplicação. As datas e as condições climáticas nas aplicações dos tratamentos estão descritas na Tabela 2.

A colheita foi realizada em 25 de fevereiro de 2015 (114 dias após a emergência) sendo avaliada, na área útil das parcelas, a produtividade de grãos (colheita das plantas com debulha das vagens e pesagem dos grãos com correção da umidade para 13\%), a massa de mil grãos (pesagem de mil grãos a partir da amostra de produtividade, com correção da umidade para 13\%), o número de vagens por planta (contagem do número de vagens em cinco plantas), e a altura de plantas e de inserção da primeira vagem (medição do colo ao último trifólio completamente expandido e à 
inserção da primeira vagem, respectivamente), além da população final (contagem do número de plantas).

Todos os dados foram submetidos à análise de variância com emprego do teste F. Uma vez constatado efeito significativo, foi empregado o teste de Scott Knott a 5\% de probabilidade para comparação das médias dos tratamentos.

\section{Resultados e discussão}

O potencial do uso dos bioestimulantes pode ser comprovado pelos resultados obtidos com o MC Extra $^{\circledR}\left(\mathrm{T}_{2}\right)$ e Megafol ${ }^{\circledR}\left(\mathrm{T}_{3}\right)$, ambos aplicados quando a soja estava com cinco trifólios desenvolvidos $\left(\mathrm{V}_{5}\right)$, além do MC Extra ${ }^{\circledR}$ e Megafol $^{\circledR} \mathrm{em} \mathrm{V}_{5}$ e R , respectivamente (Tabela 3 ). Destaca-se também o uso do MC Extra ${ }^{\circledR}$ em $V_{5}$ e $R_{5}\left(T_{6}\right)$, MC Extra $^{\circledR}$

Tabela 2. Condições climáticas nas respectivas épocas de aplicação dos tratamentos do experimento de bioestimulantes derivados de Ascophyllum nodosum associados ao glyphosate nas características agronômicas da soja $\mathrm{RR}^{\circledR}$. Montividiu-GO, 2014/15.

\begin{tabular}{lccc}
\hline & $\mathbf{1}^{\mathbf{a}}$ Aplicação & $\mathbf{2}^{\mathbf{a}}$ Aplicação & $\mathbf{3}^{\mathbf{a}}$ Aplicação \\
\hline Estádio & $\begin{array}{c}\text { Cinco trifólios } \\
\text { desenvolvidos }\left(\mathrm{V}_{5}\right)\end{array}$ & Início do florescimento $\left(\mathrm{R}_{1}\right)$ & $\begin{array}{c}\text { Início do enchimento de } \\
\text { grãos }\left(\mathrm{R}_{5}\right)\end{array}$ \\
Data & $04 / 12 / 2014$ & $20 / 12 / 2014$ & $30 / 12 / 2014$ \\
Horário da aplicação & $07: 45$ & $15: 25$ & $11: 30$ \\
Velocidade vento & 2,0 & 8,4 & 2,3 \\
$\mathrm{~T}^{\mathrm{C}} \mathrm{C}$ & 27,3 & 25,8 & 29,5 \\
Umidade relativa & 75,0 & 77,8 & 43,0 \\
\hline
\end{tabular}

Tabela 3. Valores médios e significâncias das variáveis, produtividade (PROD) e massa de mil grãos (MMG) e número de vagens totais (NVT) do experimento de bioestimulantes derivados de Ascophyllum nodosum associados ao glyphosate nas características agronômicas da soja $\mathrm{RR}^{\circledR}$. Montividiu-GO, 2014/15.

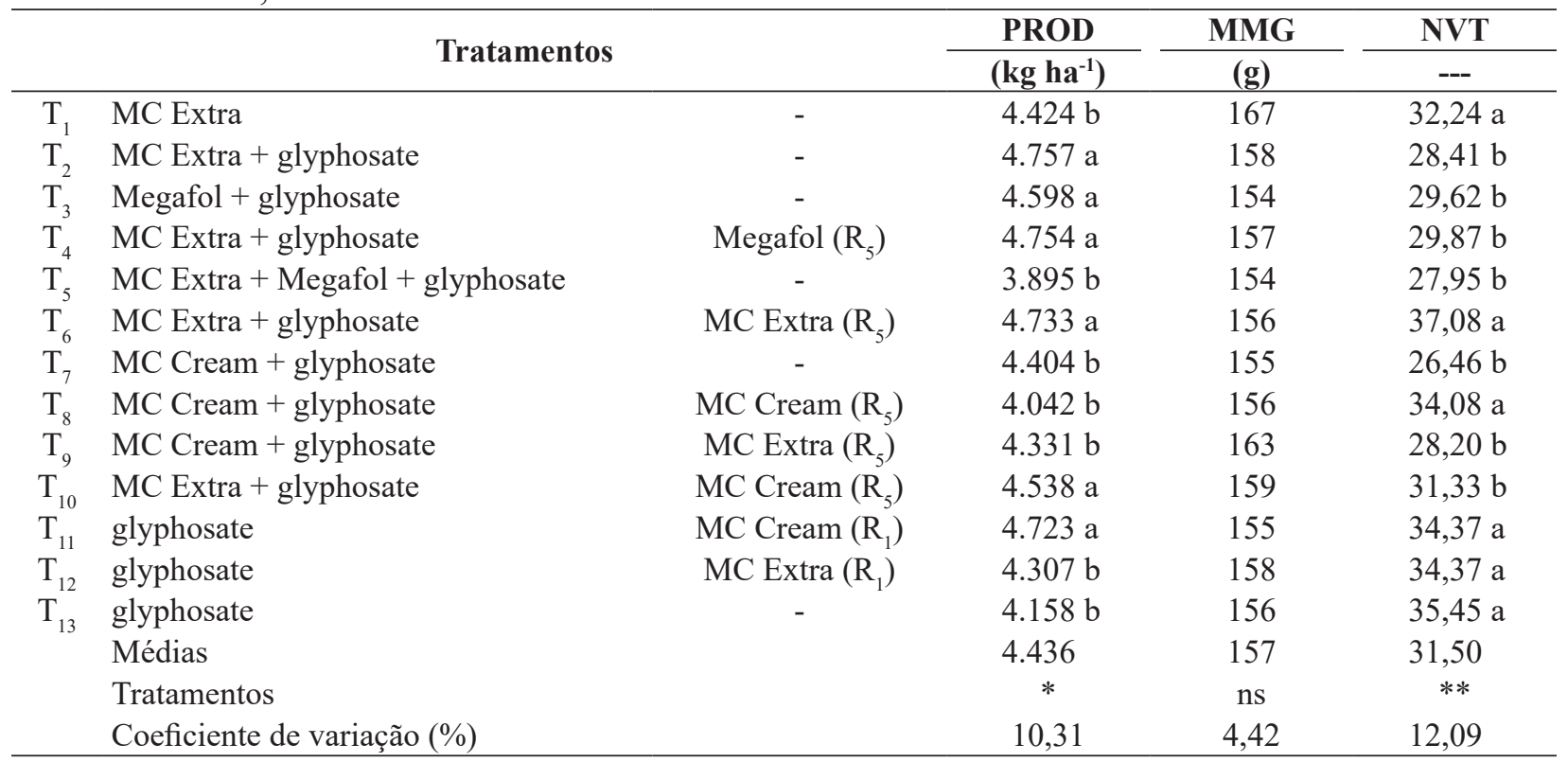

*; **; ns: Significativo a 1 e 5\% de probabilidade e não significativo, respectivamente, pelo teste F. Médias seguidas pelas mesmas letras não diferem entre si pelo teste de Scott Knott a 5\% de probabilidade. 
e MC Cream ${ }^{\circledR} \mathrm{em}_{5}$ e $\mathrm{R}_{5}$, respectivamente $\left(\mathrm{T}_{10}\right)$ e do MC Cream ${ }^{\circledR} \mathrm{em} \mathrm{R}_{1}\left(\mathrm{~T}_{11}\right)$. Todos estes tratamentos tiveram produtividade de grãos superiores ao tratamento sem bioestimulante $\left(\mathrm{T}_{13}\right)$. É importante destacar que os tratamentos $\mathrm{T}_{2}, \mathrm{~T}_{4}, \mathrm{~T}_{6}$ e $\mathrm{T}_{10}$ apresentam como semelhança a aplicação do MC Extra ${ }^{\circledR}$ com glyphosate em $V_{5}$.

$\mathrm{O}$ bioestimulante em questão possui em sua composição micronutrientes, como o ferro, cobre, zinco e manganês, que atuam como ativadores enzimáticos que incrementam a atividade metabólica da planta. A maior disponibilidade de manganês, pelo uso de bioestimulante, pode proporcionar maior síntese de clorofila, proporcionando incrementos na taxa fotossintética (Albrecht et al., 2011). O aumento das atividades enzimáticas, juntamente com a presença dos hormônios vegetais, potencializa a emissão de sinais químicos, favorecendo o processo de divisão e expansão celular, resultando assim em maior crescimento vegetativo (Taiz et al., 2017). Consequentemente, há maior síntese e translocação de metabólitos, proporcionando maiores produtividades (Povero et al., 2016), como observado no trabalho.

Adicionalmente, acredita-se que os adjuvantes presentes na formulação do Roundup Transorb ${ }^{\circledR}$ tenham contribuído com a penetração e absorção do MC $\operatorname{Extra}^{\circledR}\left(\mathrm{T}_{2}, \mathrm{~T}_{4}, \mathrm{~T}_{6} \mathrm{e} \mathrm{T}_{10}\right)$, visto que o uso desse bioestimulante sem o herbicida na calda de pulverização $\left(T_{1}\right)$ teve produtividade inferior aos tratamentos acima mencionados (Tabela 3). Neste contexto, dentre os tratamentos com o MC Extra, $\mathrm{o}_{2}$ foi o mais promissor pois envolveu apenas uma aplicação do produto (produtividade 7\% superior ao que recebeu apenas o bioestimulante em questão - $\mathrm{T}_{1}$ ). $\mathrm{O}$ sinergismo do bioestimulante com o Roundup Transorb na mesma calda de pulverização é comprovado pela semelhança das médias de produtividade entre os tratamentos com MC Extra ${ }^{\circledR}$ sem o herbicida $\left(\mathrm{T}_{1}\right.$ e $\left.\mathrm{T}_{12}\right)$ com o tratamento sem o bioestimulante $\left(\mathrm{T}_{13}\right)$.

É válido ressaltar que a penetração dos compostos nutricionais pela cutícula é lenta em função do raio iônico dos elementos. No entanto acredita-se que este processo foi potencializado pela ação dos adjuvantes contidos no herbicida. Ao quebrar a tensão superficial da gota, há maior aderência dos compostos na superfície da folha. Isto diminui consideravelmente o tempo de penetração do bioestimulante em associação com o herbicida, possivelmente em função de seus efeitos na cutícula e na permeabilidade da membrana plasmática (Mackinnon et al., 2010).

Outros efeitos benéficos do MC Extra ${ }^{\circledR}$ com o herbicida $\left(\mathrm{T}_{2}\right)$ pode ser comprovado na comparação das médias de produtividade com os demais tratamentos que envolveram a aplicação de mais de um bioestimulante. Neste sentido, a aplicação do $\mathrm{MC}$ Extra $^{\circledR} \mathrm{em} \mathrm{V}_{5}$ suprimiu o efeito dos demais bioestimulantes aplicados em estádios mais avançados de desenvolvimento da soja $\left(\mathrm{R}_{5}\right)$ (Megafol e MC Cream- $\mathrm{T}_{4}$ e $\mathrm{T}_{10}$, respectivamente) (Tabela 3).

Outro ponto interessante a se analisar no uso do MC Extra ${ }^{\circledR}$ é quanto ao fracionamento da dose utilizada no experimento. Partindo-se do princípio que aplicações em estádios avançados de desenvolvidos não promovam efeitos na produtividade da soja, a semelhança do $\mathrm{T}_{6}$ com o $\mathrm{T}_{2}$ permite inferir uma possível redução de dose do produto. Em estádios avançados de desenvolvimento, a planta de soja já definiu o porte e o número de vagens por planta, sem influência dos promotores de crescimento. O balanço de auxinas e citocinas do MC Extra foi fundamental para justificar estes efeitos, uma vez que no processo de produção do MC Extra ${ }^{\circledR}$ há redução da concentração das giberelinas, devido ao método de extração e processamento da alga (Van Oosten et al., 2017). Em função da relação desses hormônios, há maior crescimento do sistema radicular, além do estímulo ao crescimento vegetativo (Taiz et al., 2017), gerando efeitos benéficos na produtividade de grãos.

Para o MC $\mathrm{Cream}^{\circledR}$, as aplicações com glyphosate $\left(\mathrm{T}_{7}, \mathrm{~T}_{8} \mathrm{e} \mathrm{T}_{9}\right)$ apresentaram produtividades semelhantes ao tratamento sem o bioestimulante $\left(\mathrm{T}_{13}\right)$ e inferiores aos tratamentos contendo $\mathrm{MC}$ Extra $^{\circledR}$ e Megafol $^{\circledast}\left(\mathrm{T}_{2}\right.$ e $\left.\mathrm{T}_{3}\right)$ (Tabela 3). Mesmo 
a aplicação sequencial de $\mathrm{MC} \mathrm{Cream}{ }^{\circledR}\left(\mathrm{T}_{8}\right)$ ou a aplicação posterior do $\mathrm{MC}$ Extra em $\mathrm{R}_{5}\left(\mathrm{~T}_{9}\right)$ não foram suficientes para proporcionar acréscimos de produtividade de grãos da soja. Uma das hipóteses seria que o glyphosate pode ter inibido os efeitos dos componentes presentes no MC Cream ${ }^{\circledR}$.

Entretanto, a aplicação desse bioestimulante em $\mathrm{R}_{1}$, dezesseis dias após a aplicação do glyphosate $\left(\mathrm{T}_{11}\right)$, proporcionou produtividade de grãos $11 \%$ superior a aplicação somente do Roundup Transorb $\left(\mathrm{T}_{13}\right)$. Isto deve-se, possivelmente, a maior concentração de giberelinas no $\mathrm{MC}$ Cream $^{\circledR}$, induzindo a formação e pegamento de flores, proporcionando um efeito reparador da fitotoxicidade do herbicida, gerando aumento na produtividade de grãos (Povero et al., 2016).

Em relação ao Megafol ${ }^{\circledR}$, efeito positivo na produtividade de grãos foi constatado com aplicação em conjunta com Roundup Transorb $\left(\mathrm{T}_{3}\right)$ (Tabela 3). Já a associação com o herbicida e com o MC Extra na mesma calda de pulverização $\left(\mathrm{T}_{5}\right)$ não proporcionou incremento na produtividade, provavelmente devido aos efeitos do MC Extra, conforme discutido anteriormente. Em trigo já foi comprovado o efeito benéfico do Megafol ${ }^{\circledR}$ em incrementos na produtividade e no acúmulo de potássio, magnésio e cálcio e manganês, cobre e zinco nos grãos (Miroshnichenko et al., 2017).

Os bioestimulantes não influenciaram a massa de mil grãos e a altura de inserção da primeira vagem, ao contrário do número de vagens totais e altura de plantas (Tabelas 3 e 4). Os maiores valores para o número de vagens foram observados para os tratamentos com o parcelamento da dose de $M C$ Extra ${ }^{\circledR}$, em $\mathrm{V}_{5}$ e $\mathrm{R}_{5}\left(\mathrm{~T}_{6}\right)$ e do uso do $\mathrm{MC}$ Cream ${ }^{\circledR} \mathrm{em} \mathrm{R}_{1}\left(\mathrm{~T}_{11}\right)$. Destaca-se também a maior altura de plantas com uso conjunto do MC Extra e Roundup Transorb ( $\left.T_{3}\right)$ (Tabela 4), podendo ter contribuído para incrementos na produtividade de grãos da soja.

O decréscimo da síntese de clorofila, da taxa fotossintética e da imobilização de cátions (ferro,

Tabela 4. Valores médios e significâncias das variáveis alturas de plantas inicial, final e de inserção da primeira vagem (AIV), além da população de plantas (POP) do experimento de bioestimulantes derivados de Ascophyllum nodosum associados ao glyphosate nas características agronômicas da soja RR ${ }^{\circledast}$. Montividiu-GO, 2014/15.

\begin{tabular}{|c|c|c|c|c|c|}
\hline & Tratamento & & AP & AIV & POP \\
\hline & Tratament & & & & FOP \\
\hline $\mathrm{T}_{1}$ & MC Extra & - & $62,54 \mathrm{~b}$ & 11,95 & $398.333 \mathrm{~b}$ \\
\hline $\mathrm{T}_{2}$ & MC Extra + glyphosate & - & 67,79 a & 13,25 & $395.625 \mathrm{~b}$ \\
\hline $\mathrm{T}_{3}^{2}$ & Megafol + glyphosate & - & $60,91 \mathrm{~b}$ & 11,21 & $430.625 \mathrm{a}$ \\
\hline $\mathrm{T}_{4}^{3}$ & MC Extra + glyphosate & Megafol $\left(R_{5}\right)$ & $63,12 \mathrm{~b}$ & 12,04 & $415.000 \mathrm{a}$ \\
\hline $\mathrm{T}_{5}$ & MC Extra + Megafol + glyphosate & & $63,79 \mathrm{~b}$ & 12,58 & $416.875 \mathrm{a}$ \\
\hline $\mathrm{T}_{6}$ & MC Extra + glyphosate & MC Extra $\left(R_{5}\right)$ & $64,00 \mathrm{~b}$ & 11,62 & $406.250 \mathrm{~b}$ \\
\hline $\mathrm{T}_{7}^{0}$ & MC Cream + glyphosate & - & $63,25 \mathrm{~b}$ & 12,66 & $396.250 \mathrm{~b}$ \\
\hline $\mathrm{T}_{8}$ & MC Cream + glyphosate & $\operatorname{MC~Cream~}\left(\mathrm{R}_{5}\right)$ & 70,83 a & 11,91 & $405.000 \mathrm{~b}$ \\
\hline $\mathrm{T}_{9}^{8}$ & MC Cream + glyphosate & $\mathrm{MC} \operatorname{Extra}\left(\mathrm{R}_{5}\right)$ & $60,79 \mathrm{~b}$ & 12,37 & $425.625 \mathrm{a}$ \\
\hline $\mathrm{T}_{10}$ & MC Extra + glyphosate & MC Cream $\left(\mathrm{R}_{5}\right)$ & $64,33 \mathrm{~b}$ & 12,41 & $415.625 \mathrm{a}$ \\
\hline $\mathrm{T}_{11}$ & glyphosate & $\operatorname{MC~Cream~}\left(\mathrm{R}_{1}\right)$ & $65,37 \mathrm{~b}$ & 12,25 & $401.250 \mathrm{~b}$ \\
\hline $\mathrm{T}_{12}$ & glyphosate & MC Extra $\left(R_{1}\right)$ & $61,74 \mathrm{~b}$ & 11,29 & $405.000 \mathrm{~b}$ \\
\hline $\mathrm{T}_{13}$ & glyphosate & - & $63,58 \mathrm{~b}$ & 11,95 & $418.750 \mathrm{a}$ \\
\hline & Médias & & 64,00 & 12,11 & 410.016 \\
\hline & Tratamento & & $* *$ & ns & $*$ \\
\hline & Coeficiente de variação (\%) & & 5,40 & 7,72 & 4,72 \\
\hline
\end{tabular}

*; **; ns: Significativo a 1 e $5 \%$ de probabilidade e não significativo, respectivamente, pelo teste $\mathrm{F}$. Médias seguidas pelas mesmas letras não diferem entre si pelo teste de Scott Knott a 5\% de probabilidade. 
manganês, zinco e cobre) pelo glyphosate pode ter ocasionado limitação no crescimento da soja, gerando plantas de menor porte (Constantin et al., 2016). Como o aumento na altura de plantas depende da energia suprida pela fotossíntese para sintetizar compostos carbônicos, diminuições na assimilação do $\mathrm{CO}_{2}$ levam à redução no acúmulo de carboidratos e de biomassa nas plantas (Magalhães Filho et al., 2008). Neste sentido, acredita-se que a ação dos adjuvantes, presentes na formulação do Roundup Transorb, proporcionou maior penetração e absorção do MC Extra ${ }^{\circledR}$, estimulando assim as atividades fisiológicas da planta (Cunha et al., 2017).

Dessa forma foi possível constar, em determinadas condições, que os bioestimulantes derivados de Ascophyllum nodosum tem a capacidade de promoverem incrementos benéficos nas características agronômicas da soja. Em determinados casos e ao contrário do $\mathrm{MC}$ Cream $^{\circledR}$, a associação com Roundup Transorb para manejo de plantas daninhas (emprego do MC Extra ${ }^{\circledR}$ e Megafol $^{\circledR}$ ) beneficia a soja quando o objetivo é o aumento de produtividade de grãos. Além disto, o aumento dessa variável pelo uso dos bioestimulantes foi atribuída a mudanças nas características morfofisiológicas na planta de soja.

Devido a composição distinta dos bioestimulantes, é interessante atentar para o emprego adequado desses produtos, principalmente no que se refere a doses, estádios de aplicação e associação com defensivos, como o glyphosate. Uma vez identificados o adequado posicionamento, os bioestimulantes proporcionam efeitos benéficos para a cultura da soja, possibilitando assim rentabilidade com o cultivo de soja.

\section{Conclusões}

O MC Extra ${ }^{\circledR}$ e o Megafol ${ }^{\circledR}$ proporcionaram incrementos na produtividade de grãos em associação com Roundup Transorb com aplicação no estádio $\mathrm{V}_{5}$.

O MC Cream ${ }^{\circledR}$ proporcionou maior produtividade de grãos aos quinze dias após a aplicação do Roundup Transorb.

\section{Referências}

Albrecht, L.P.; Braccini, A.L.; Scapim, C.A.; Ávila, M.R.; Albrecht, A.J.P.; Ricci, T.T. Manejo de biorregulador nos componentes de produção e desempenho das plantas de soja. Bioscience Journal, v.27, n.6, p.865-876, 2011.

Beckie, H.J. Herbicide-resistant weed management: focus on glyphosate. Pest Management Science, v.67, n.9, p.1037-1048, 2011.

Bueno, A.; Moreira, A.; Balbinot Junior, A.A.; Carvalho, C.; Gazzoni, D.; Marcelino-Guimarães, F.C.; Neumaier, N. Tecnologias de produção de soja: Região Central do Brasil 2014. Londrina: EMBRAPA/CNPSO, 2014.

Brasil. Ministério da Agricultura, Pecuária e Abastecimento. CONAB - Companhia Brasileira de Abastecimento. $\mathbf{1 0}^{\circ}$ Levantamento - Safra 2017/18. Disponível em: www.conab.gov.br/. Acesso em: 10 jul. $2018 \mathrm{a}$.

Brasil. Ministério da Agricultura Pecuária e Abastecimento. Soja. Disponível em: $<$ http:// www.agricultura.gov.br $>$. Acesso em: 10 jul. de 2018b.

Constantin, J.; Oliveira Junior, R.S.; Gheno, E.A.; Biffe, D.F.; Braz, G.B.P.; Weber, F.; Takano, H.K. Prevention of yield losses caused by glyphosate in soybeans with biostimulant. African Journal of Agricultural Research, v.11, n.18, p.16011607, 2016.

Coutinho, C.F.B.; Mazo, L.H. Metallic complex with glyphosate: a review. Quimica Nova, v.28, n.6, p.1038-1045, 2005.

Cunha, J.P.A.R.; Alves, G.S.; Marques, R.S. Tensão superficial, potencial hidrogeniônico e condutividade elétrica de caldas de produtos fitossanitários e adjuvantes. Revista Ciência Agronômica, v.48, n.2, p.261-270, 2017.

Craigie, J.S. Seaweed extract stimuli in plant science and agriculture. Journal of Applied Phycology, v.23, n.3, p.371-393, 2011. 
Du Jardin, P. Plant biostimulants: definition, concept, main categories and regulation. Scientia Horticulturae, v.196, p.3-14, 2015.

Dvoranen, E.C.; Oliveira Junior, R.S.; Constantin, J.; Cavalieri, S.D.; Blainski, E. Nodulação e crescimento de variedades de soja RR sob aplicação de glyphosate, fluazifopp-butyl e fomesafen. Planta Daninha, v.26, n.3, p.619-625, 2008.

Ferreira, L.R.; Silva, A.G.; Simon, G.A.; Teixeira, I.R.; Sena, M.P.D. Glyphosate em pós-emergência na soja Roundup Ready. Revista Brasileira de Herbicidas, v.12, n.2, p.151-161, 2013.

Gava, R. Os efeitos do estresse hídrico na cultura da soja (Glycine Max, (L.) Merrill.). 2014. 123 f. Tese (Doutorado em Irrigação e Drenagem) Escola Superior de Agricultura Luiz de Queiroz, Universidade de São Paulo, Piracicaba, 2014.

Gazziero, D.L.P. Soja transgênica: o que muda no manejo das plantas daninhas. In: Congresso Brasileiro de Soja, 2006, Londrina. Anais... Londrina: EMBRAPA/CNPSO, 2006. p. 143-146.

Khan, A.S.; Ahmad, B.; Jaskani, M.J.; Ahmad, R.; Malik, A.U. Foliar application of mixture of amino acids and seaweed Ascophylum nodosum extract improve growth and physicochemical properties of grapes. International Journal of Agriculture and Biology, v.14, n.3, p.383-388, 2012.

López-Ovejero, R.F.; Penckowski, L.H.; Podolam, M.J.; Carvalho, S.J.P.; Christoffoleti, P.J. Alternativas de manejo químico da planta daninha Digitaria insularis resistente aos herbicidas inibidores da ACCase na cultura da soja. Planta Daninha, v.24, n.2, p.407-414, 2006.

Mackinnon, S.L.; Hiltz, D.; Ugarte, R.; Craft, C.A. Improved methods of analysis for betaines in Ascophyllum nodosum and its commercial seaweed extracts. Journal of Applied Phycology, v.22, n.4, p.489-494, 2010.

Magalhaes Filho, R.; Rios do Amaral, L.; Fávero São Pedro Machado, D.; Medina, C.L.; Caruso, M.E. Deficiência hídrica, trocas gasosas e crescimento de raízes em laranjeira 'Valência' sobre dois tipos de porta-enxerto. Bragantia, v.67, n.1, p.75-82, 2008.

Merotto, A.; Wagner, J.; Meneguzzi, C. Effects of Glyphosate and foliar application of micronutrients in transgenic soybean. Bioscience Journal, v.31, n.2, p.499-508, 2015.

Miroshnichenko, I.M.; Makoveychuk, T.I.; Mykhalska, L.M.; Schwartau, V.V. Changes in the elemental composition of winter wheat plants caused by the action of Megafol and retardants. Regulatory Mechanisms in Biosystems, v.8, n.3, p.403-409, 2017.

Procópio, S.O.; Santos, J.B.; Jacques, R.J.S.; Kasuya, M.C.M.; Silva, A.A. Crescimento de estirpes de Bradyrhizobium sob influência dos herbicidas glyphosate potássico, fomesafen, imazethapyr e carfentrazone-ethyl. Revista Ceres, v.51, n.294, p.179-188, 2004.

Povero, G.; Mejia, J.F., Di tommaso, D.; Piaggesi, A.; Warrior, P. A systematic approach to discover and characterize natural plant biostimulants. Frontiers in Plant Science, v.7, n.435, p.1-9, 2016.

Santos, J.B.; Ferreira, E.A.; Reis, M.R.; Silva, A.A.; Fialho, C.M.T.; Freitas, M.A.M. Avaliação de formulações de glyphosate sobre soja Roundup Ready. Planta Daninha, v.25, n.1, p.165-171, 2007.

Sharma, S.H.S.; Lyons, G.; Mcroberts, C.; Mccall, D.; Carmichael, E.; Andrews, F.; et al. Biostimulant activity of brown seaweed species from Strangford Lough: compositional analyses of polysaccharides and bioassay of extracts using mung bean (Vigno mungo L.) and pak choi (Brassica rapa chinensis L.). Journal of Applied Phycology, v.24, p.1081-1091, 2012.

Taiz, L.; Zeiger, E.; Moller, I.M.; Murphy, A. Fisiologia e desenvolvimento vegetal. 6.ed. Porto Alegre: Artmed Editora, 2017. 888p.

United States. USDA - United States Department of Agriculture. Soybeans: World Supply and 
Distribution 2018. Disponível em: <apps.fas. to glyphosate in glyphosate-resistant soybean. usda.gov/psdonline/>. Acesso em: 10 jul. de Crop Protection (Guildford, Surrey), v.26, n.3, 2018. p.370-376, 2007.

Van Oosten, M.J.; Pepe, O.; Pascale, S.; Silletti, S.; Maggio, A. The role of biostimulants and bioeffectors as alleviators of abiotic stress in crop plants. Chemical and Biological Technologies in Agriculture, v.4, n.1, p.5, 2017.

Zobiole, L.H.S.; Oliveira, R.S.; Huber, D.M.; Constantin, J.; Castro, C.; Oliveira, F.A.; Oliveira, A. Glyphosate reduces shoot concentration of mineral nutrients in glyphosateresistant soybeans. Plant and Soil, v.328, n.1, p.57-69, 2010.

Vandenkoornhuyse, P.; Quaiser, A.; Duhamel, M.; Le Van, A.; Dufresne, A. The importance of the microbiome of the plant holobiont. The New Phytologist, v.206, n.4, p.1196-1206, 2015.

Zobiole, L.H.S.; Oliveira Junior, R.S.; Constantin, J.; Oliveira Junior, A.; Castro, C.; Oliveira, F.A.; Romagnoli, L.M. Acúmulo de nutrientes em soja convencional e soja RR em diferentes tipos de Zablotowicz, R.M.; Reddy, K.N. Nitrogenase activity, nitrogen content, and yield responses controle de planta daninha. Planta Daninha, v.30, n.1, p.75-85, 2012. 